\title{
Proposed SMART Traffic Control Signal in Brunei Darussalam
}

\author{
Bibi Rawiyah Mulung*, Andino Maseleno \\ Electrical Engineering Department, Faculty of Engineering, Institut Teknologi Brunei, \\ Negara Brunei Darussalam \\ Computer Science Program, Universiti Brunei Darussalam, \\ Negara Brunei Darussalam \\ *Corresponding author, e-mail: rawiyah3293@hotmail.co.uk
}

\begin{abstract}
This paper presents proposed SMART (Systematic Monitoring of Arterial Road Traffic Signals) traffic control signal in Brunei Darussalam. Traffic congestion due to stops and delays at traffic light signals has much been complained about in Brunei Darussalam as well as across the world during the recent years. There are primarily two types of traffic signal controls in Brunei Darussalam. The most common one is the fixed or pre-timed signal operation traffic light and the other one is the actuated signal operation traffic light. Although the actuated signal control is more efficient than the fixed or pre-fixed signal control in the sense that it provides fewer stops and delays to traffic on the major arteries, the best option for Brunei Darussalam would be to introduce smart traffic control signal. This type of traffic signal uses artificial intelligence to take the appropriate action by adjusting the times in real time to minimise the delay in the intersection while also coordinating with intersections in the neighbourhood. SMART Signal simultaneously collects event-based high-resolution traffic data from multiple intersections and generates real-time signal performance measures, including arterial travel time, number of stops, queue length, intersection delay, and level of service. In Brunei Darussalam, where we have numerous intersections where several arterial roads are linked to one another, The SMART signal traffic control method should be implemented.
\end{abstract}

Keywords: smart traffic, traffic congestion control, Brunei Darussalam

Copyright $\odot 2015$ Institute of Advanced Engineering and Science. All rights reserved.

\section{Introduction}

Brunei Darussalam, officially the Nation of Brunei, the Abode of Peace is a sovereign state located on the north coast of the island of Borneo, in Southeast Asia [1]. Apart from its coastline with the South China Sea, it is completely surrounded by the state of Sarawak, Malaysia; and it is separated into two parts by the Sarawak district of Limbang. The two main sections under the Department of Roads which are responsible for the planning, construction and management of the road network system in Brunei Darussalam are Road Construction Section (SRC) and Road Management Road Maintenance (SRM) [2].

The Road Construction Section (SRC) is responsible for developing new roads, highways, slip roads, junctions, roundabouts, bridges and flyovers are planned and implemented [2]. It is also responsible for the access to the rural communities and also the Public Works Department had constructed (as of December 2010): 26 Flyovers, 84 Signalised Traffic Lights and 8 Roundabouts [2]. After the projects are completed by the Road Construction Section (SRC), the maintenance of the infrastructures on the road, mainly the road itself, bridges, road-side drains, traffic lights are taken care of by the Road Maintenance Section (SRM). The Road Management Road Maintenance (SRM) overlooks the Traffic Light Management Unit. The Area Traffic Control (ATC) was established in 2002 and was renamed Traffic Management Centre (TMC) in 2012. It currently manages traffic light junctions.

Some researchers have studied smart traffic control system. Hasan [3] proposed a method for determining traffic congestion on roads using image processing techniques and a model for controlling traffic signals based on information received from images of roads taken by video camera. Nayak et al. [4] describes a method to solve the problem of invisibility of traffic signal caused by huge vehicles blocking the view, prevent traffic congestion at toll gates and give advanced collision warning to the drivers. Colotta et al. [5] proposed smart traffic light 
junction management using wireless sensor networks. In Brunei Darussalam, proposed traffic control signal simultaneously collects event-based high-resolution traffic data from multiple intersections and generates real-time signal performance measures, including arterial travel time, number of stops, queue length, intersection delay, and level of service.

\section{Traffic Signal Control}

There are primarily two types of traffic signal control in Brunei Darussalam: The fixed or pre-timed signal operation and the actuated signal operation. Pre-timed traffic controllers perate in a predetermined and regularly repeated sequence of signal indications [6]. The operation of the pre-timed traffic signal is such that each time, a signal phase or traffic movement is serviced in a programmed sequence that is repeated throughout the day. Main street traffic receives a fixed amount of green time followed by the amber and red clearance intervals. The same interval timing is the repeated for the minor or side street. The amount of time it takes to service all conflicting traffic movements is referred to as the cycle length. The signal timings and cycle lengths may vary by time of day to reflect changes in traffic volumes and patterns. During peak traffic periods for example, cycle lengths may range from 90-128 seconds to accommodate heavier volumes, particularly on the busier arterial roadways. During off peak times of day, cycle lengths are reduced as traffic volumes are much lighter and therefore not much of green time is required to effectively service all movements.

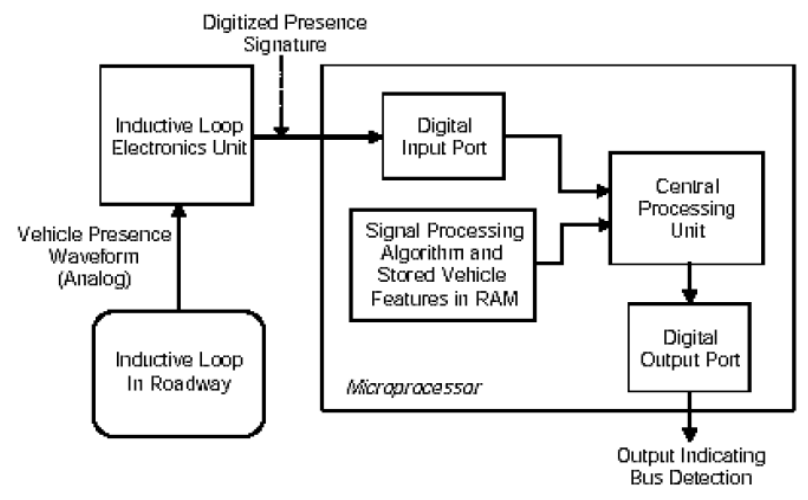

Figure 1. Simplified diagram of actuated traffic signal control system

Actuated signal control differs from pre-timed in that it requires actuation by a vehicle or pedestrian in order for certain phases or traffic movements to be serviced. Figure 1 shows simplified diagram of actuated traffic signal control system. Actuation is achieved by vehicle detection devices and pedestrian push buttons. The most common method of detecting vehicles is to install inductive loop wires in the pavement at or near the painted stop bar. Video detection is also used at select locations. Actuated signals consist of two types: semi-actuated and fullyactuated. In the semi-actuated system, vehicle loops detectors are installed on the minor street approaches and push buttons are provided for pedestrians wanting to cross the major roadway. The traffic signals remain green on the major roadway until either a cross street vehicle is detected or a pedestrian pushes the button. When this occurs, a call is sent to the traffic signal controller at appropriate time in the cycle. The main street green will terminate and time its clearance intervals before the minor street is serviced. If the side street is servicing vehicle demand only, a minimum green of 5-7 seconds are provided which can extend up to a preset maximum provided additional vehicles are being detected. After the last vehicle passes over the detector loop or the preset maximum green time has been reached, the signals will return to a green state on the main street. In the fully-actuated system, vehicle detector loops and pedestrian push buttons are installed on all approaches. All signal phases including left turn arrows have preset minimum and maximum greens and will be serviced on demand only. Pedestrians much activate the push button in order to receive the indications. A single press of the button locks the call in the controllers memory that a pedestrian has requested service. 


\section{System Model and Problem Statement}

In the fixed or pre-timed signal operation, both arrivals at the signal and departures of vehicles are assumed to be at strictly regular intervals. This type of control gives a fairly good prediction of delays when the traffic is light. However, because it predicts that queues will always be exhausted at the end of the green phase, provided that the rate of arrival does not exceed the capacity of the junction, delays are seriously underestimated when arrival rates are fairly high. Figure 2 shows an urban scenario using actuated traffic signal control system. Although actuated signal control provides greater efficiency compared to pre-timed signals by servicing cross street traffic and pedestrians only when required, fully-actuated signals are most efficient at isolated locations where coordination with adjacent signals is not a concern and where the intersecting roadways have similar traffic volumes. These limitations give rise to road congestion which leads to several issues such as:

1) Road accidents: According to statistics from the Royal Brunei Police Force, 24 road fatalities were recorded in the year 2013 up to August 31 [7]. Causes of these road fatalities differ and usually involve careless drivers who go beyond the speed limit or use their mobile phones while driving. However, another factor is beating the red light and being inconsiderate and insensitive to other road users. The drivers often indulge in such actions due to irritation or impatience while waiting for the traffic lights to turn green.

2) Impact on public health: Air pollution from traffic congestion in 83 countries of the nation's largest urban areas contribute to more than 2,200 premature deaths annually, costing the health system at least $\$ 18$ billion, according to a study by Harvard School of Public Health (HSPH) [8].

3. Impact on country's economy: The human hours lost in traffic congestion can impact a country's economic productivity as evidenced by a report on the notorious congestion in Bangladesh's capital [9] that found workers spent 3.2 million business hours stuck in their cars, biting nearly $\$ 3$ million out of the $\$ 100$ million economy. A 2013 report from the Texas A\&M Transportation Institute found that the financial cost of congestion in the United States in 2011 was $\$ 121$ billion, translating to $\$ 818$ per U.S. commuter.

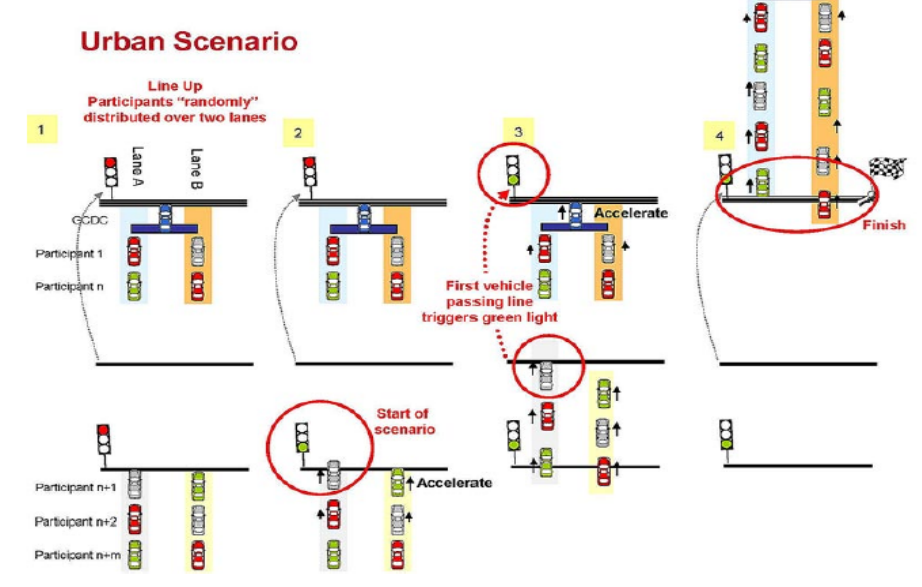

Figure 2. An urban scenario using actuated traffic signal control system

\section{Methods and Solutions}

SMART Signal (Systematic Monitoring of Arterial Road Traffic Signals) simultaneously collects event-based high resolution traffic data from multiple intersections and generates realtime signal performance measures, including arterial travel time, number of stops, queue length, intersection delay and level of service. SMART Signal simulates the motions of a virtual probe vehicle along the arterial road. As the virtual probe moves, it can change its own state in response to the state of traffic around it by accelerating, decelerating or maintaining a constant speed at each time step as it encounters queues, traffic signals and changes in traffic density. The proposed model requires a systematic approach for collecting and archiving high-resolution 
traffic data and the development of procedures and methodologies for extracting a rich set of performance measures from high resolution data.

\subsection{System Modelling}

The SMART Signal has three major components namely the event-based data collection system, performance measure calculation system and user interface. Figure 3 shows SMART signal system modelling.

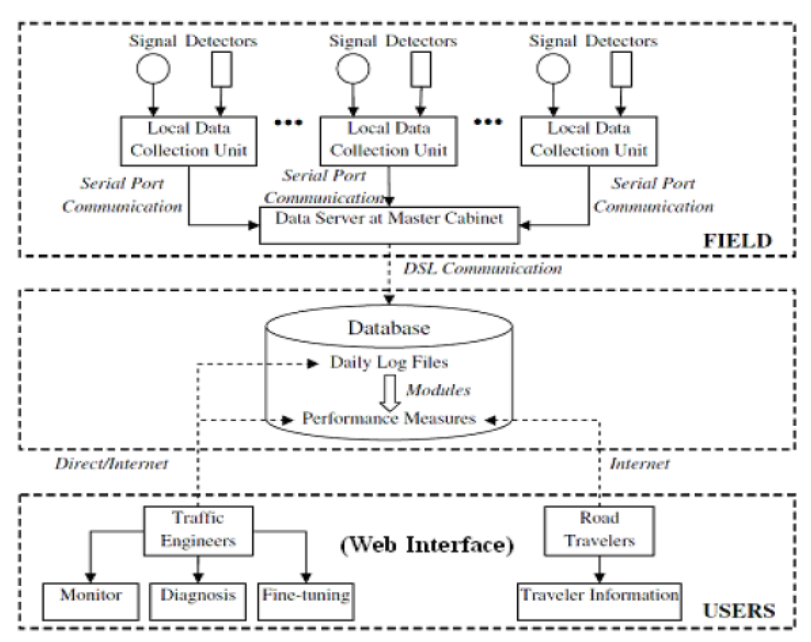

Figure 3. SMART signal system modeling

The first box shows the Data Collection System which is implemented in the field. Two types of data, the signal event and detector are collected by the system and stored in a log file every day. Data in different intersections is transmitted to the master cabinet by using serial ports of computers and then sent back to the database at the Traffic Lab. In the second box, a rich set of performance measures are generated in the database, including both intersection level and arterial level, such as volume, queue, delay, travel time and other relevant information. Users can access information such as the volume in one approach of one intersection in a certain time, as illustrated in the third box.

\subsection{Data Collection System}

Two types of event signals, namely vehicle actuation events and signal phase change events, would be captured by the Data Collection System. A detector call, or actuation, indicates a vehicle arriving event and that the vehicle demands service for a particular motion. Signal phase changes are also regarded as an event. The signal event and detector event are acquired separately from the data collection units located in the traffic signal cabinets. Vehicle actuation events are captured by inductive loop detectors, whose inductance can be changed when a vehicle passes by. Detection units in the cabinet convert the change of inductance to the change of voltage and a typical digital signal is sent out to the back panel after modulation which will be used by the traffic controller in the cabinet. Signal status is determined by the controller and a high/low digital control signal is sent out to switch the traffic lights. The scope is expanded to a group of consecutive intersections on arterials. At each intersection, an industrial PC with a data acquisition card is installed, and event data collected at each intersection are transmitted to the data server in the master controller cabinet through the existing communication line between signalised intersections.

The elements of data collection at each intersection can essentially be divided into two categories: the existing traffic signal elements and the additional data collection elements. Existing elements include the vehicle detection units, the traffic signal controller, and the back panel of the traffic cabinet. The additional components include a Traffic Controller Interface Device (CID), Traffic Event Recorder software program and a Traffic Log Database. The CID is 
a piece of hardware which physically connects the existing traffic signal components with an industrial computer installed in the cabinet on site. As the vehicle actuation events and signal status change, events will be finally represented by the high-low voltage changes on the back panel, digital data acquisition card can be used to capture these events. Figure 4 shows traffic data collection component.

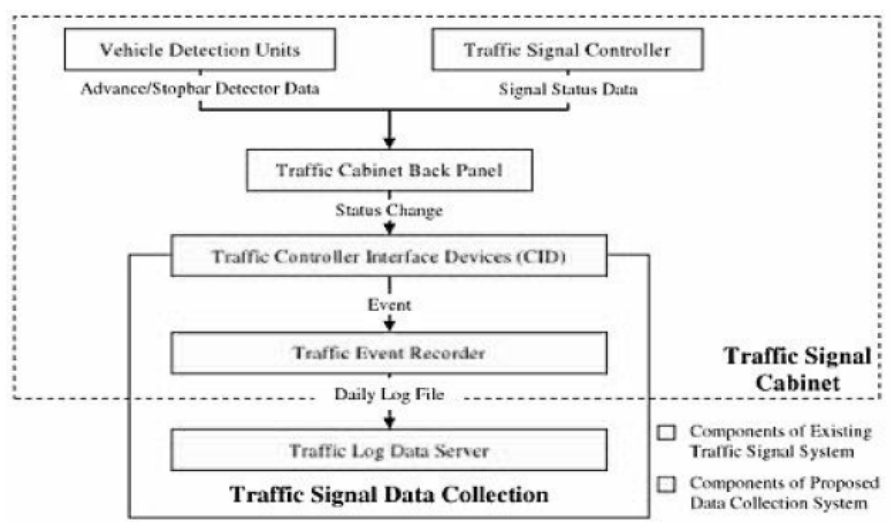

Figure 4. Traffic data collection Component

\subsection{Data Processing}

The first module in this procedure is called Data Verification, which examines all the raw records to see whether there are some errors in the data set. The second module is the preprocessing module, which generates some basic measures from the raw data. The preprocessed data has 4 groups, in terms of volume, occupancy, signal state and timing plan, and all the future performance measures are based on these pre-processed data. The third level of the data processing is the performance measure calculation. In this part, aggregated volume, delay, queue size, queue length, travel time, etc are derived from pre-processed data using different algorithms. Finally, the generated measures can be visualised and published through various techniques for road users and traffic engineers. Based on the different types of performance measures, diagnosis and fine-tune of traffics, signals can be achieved. Figure 5 shows flow chart of data process.

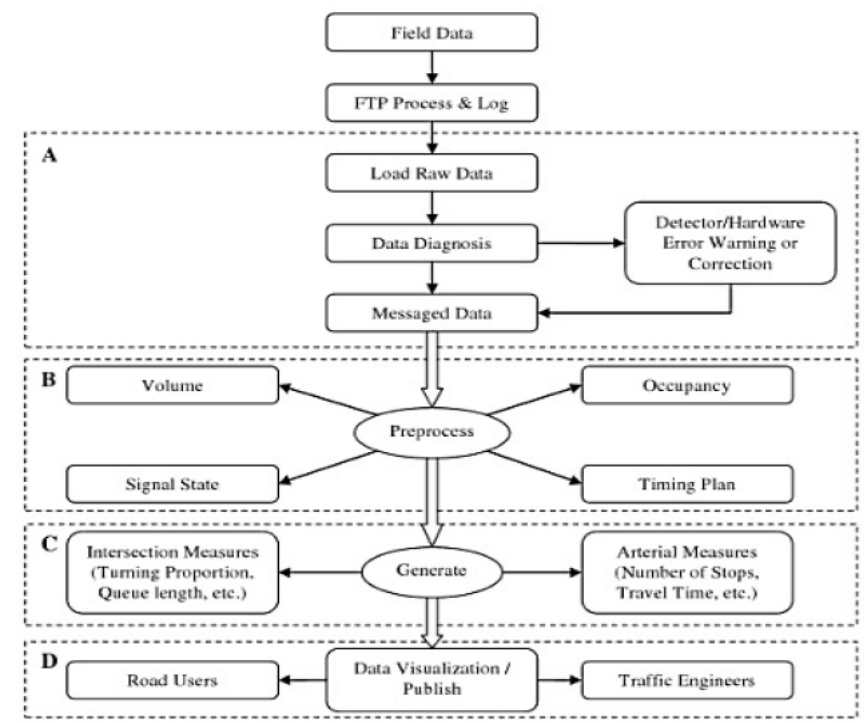

Figure 5. Flow chart of data process 


\subsection{Arterial Performance Measure}

The arterial performance measurement emphasised on is travel time estimation. Travel time is one of the most important arterial performance measures for evaluating the operation of traffic network and accurate and reliable travel time information becomes increasingly important for traffic engineers. Travel time is also one of the most understood measures for road users, helping them make informed decisions on travel choices, hence avoid unnecessary delay. The high-resolution event-based detector and signal status data are obtained as described earlier. The availability of time-stamped signal status and loop detector data essentially allows us to recon struct the history of traffic signal events along the arterial street. The time-dependent travel time is estimated by tracing the virtual probe and determining its next manoeuvre based on estimated traffic states. At each time step, the virtual probe has three possible manoeuvres: acceleration, deceleration and constant speed. The manoeuvre decision is determined by its own status and its surrounding traffic conditions. State variables of the virtual probe include its position, speed and acceleration rate. Surrounding traffic states include the status of the queue ahead of the virtual probe and the signal status. The traffic states are examined to make manoeuvre selection, and thus determine the speed and position of the probe in the next time step. All the traffic states can be calculated based on the availability of event-based traffic data at the intersections. The step-by-step manoeuvre selection continues until the virtual probe reaches the destination and the difference between the starting time and the ending time is the arterial travel time.

\section{Conclusion}

The SMART-Signal traffic control method has proven its effectiveness in a study conducted by associate professor Henry Liu of the University of Minnesota's Department of Civil Engineering to improve traffic management on urban arterial corridors. The system is now deployed at more than 100 intersections on major arterial corridors in Minnesota and Pasadena, California. In 2011, the University of Minnesotas Office of Technology Commercialization signed a licensing agreement with start-up company SMART Signal Technologies Inc. to ommercialize the system. Funding and in-kind support for the SMART Signal system have been provided by the Minnesota Department of Transportation, the ITS Institute, the Minnesota Local Road Research Board, Hennepin County, and the National Cooperative Highway Research Program. In Brunei Darussalam, we have the Lambak Kanan I/S situated in Berakas. This intersection leads to several destinations namely Jalan Penghubung Berakas: Lambak Kanan Housing and Industrial Estates, Salambigar, Tanah Jambu, Madang, Manggis, Brunei International Airport, and Bandar Seri Begawan. We also have another example of a complex intersection namely the Bukit Panggal I/S situated in the district of Tutong. The roads emerging from this specific intersection lead to alan Bukit Panggal (southwest-bound exit only): Bukit Panggal, Luagan Dudok, Sinaut, Lamunin, Jalan Bukit Panggal (northeast-bound exit only): Bukit Panggal. We have several more complex intersections like these in Brunei Darussalam. The proposed model could therefore be implemented in Brunei Darussalam to overcome the problems actually being caused by the fixed or pre-timed signal operation traffic light and the actuated signal operation traffic light. In Brunei Darussalam, where we have numerous intersections where several arterial roads are linked to one another, The SMART-Signal traffic control method should be implemented.

\section{References}

[1] Wikipedia. Brunei. http://en.wikipedia.org/wiki/Brunei. 2015.

[2] Public Works Department. Road Services. http://www.pwd.gov.bn, Negara Brunei Darussalam, 2015.

[3] Hasan MM. Smart traffic control system with application of image processing techniques. International Conference on Informatics, Electronics \& Vision (ICIEV). Dhaka, Bangladesh. 2014: 1-4.

[4] Nayak RR, Sahana SK, Bagalkot AS, Soumya M, Roopa J, Govinda RM, Ramavenkateswaran N. Smart traffic congestion control using wireless Communication. International Journal of Advanced Research in Computer and Communication Engineering. 2013; 2(9).

[5] Collotta M, Giuffre T, Pau G, Scata G. Smart Traffic Light Junction Management Using Wireless Sensor Networks. WSEAS Transactions on Communications. 2014; 13.

[6] Kurniawan F, Dermawan D, Dinaryanto O, Irawati M. Pre-Timed and Coordinated Traffic Controller Systems Based on AVR Microcontroller. TELKOMNIKA. 2014; 12(4): 787-794. 
[7] Royal Brunei Police Force. Statistics of Road Accidents. Negara Brunei Darussalam. 2013.

[8] Harvard School of Public Health. Emissions from traffic congestion may shorten lives. http://www.hsph.harvard.edu/news/hsph-in-the-news/air-pollution-traffic-levy-vonstackelberg/. Boston, USA. 2015.

[9] Smith C. Green Idea: Self-organizing Traffic Signals. Our World. United Nations University. Tokyo, Japan. 2010. 